Check for updates

Cite this: RSC Adv., 2017, 7, 34517

\title{
Dynamic electrical behaviour of a thermoresponsive polymer in well-defined poly( $N$-isopropylacrylamide)-grafted semiconductor devices $\dagger$
}

\author{
Tsukuru Masuda, (D) $\ddagger^{\mathrm{a}}$ Taira Kajisa, (DD ${ }^{\mathrm{b}}$ Aya Mizutani Akimoto, (D) a Akane Fujita, ${ }^{a}$ \\ Kenichi Nagase, (D) ${ }^{c}$ Teruo Okano, ${ }^{c}$ Toshiya Sakata (D) *a and Ryo Yoshida (D) *a
}

Received 23rd May 2017

Accepted 4th July 2017

Herein, a thermoresponsive polymer, poly( $\mathrm{N}$-isopropylacrylamide) (PNIPAAm) was grafted on a $\mathrm{Ta}_{2} \mathrm{O}_{5}$ gate surface by surface-initiated atom transfer radical polymerization. We found that the phase transition behaviour from swelling state to deswelling state in response to temperature change was electrically detected in real time by using the PNIPAAm-grafted gate FET.

rsc.li/rsc-advances

structure on a substrate. The densely packed PNIPAAm inter-

Functional soft interfaces modified with stimuli-responsive polymers have attracted considerable attention in the fields of biomaterials and biosensors. They can change their physicochemical properties in response to external environmental changes (temperature, light, $\mathrm{pH}$, chemicals) ${ }^{1-4}$ and have been investigated for use in various applications including actuators, wettability control, lubrication control, and controlled release. ${ }^{5-8}$ Among the various functional polymers, poly $(N$-isopropylacrylamide) (PNIPAAm) is one of the most widely studied thermoresponsive polymers. PNIPAAm exhibits hydrophilic and hydrophobic properties on the opposite sides of the lower critical solution temperature (LCST), which is $32{ }^{\circ} \mathrm{C}$ in aqueous media. ${ }^{9}$ Thanks to the thermo-responsivity in the form of swelling and deswelling, PNIPAAm-modified devices have been studied for various applications including flow control, as the stationary phase in chromatography, and cell culture dishes. ${ }^{10-12}$

In the past few decades, various PNIPAAm grafting techniques have been investigated to design thermoresponsive interfaces. ${ }^{11-15}$ Among them, surface-initiated atom transfer radical polymerization (SI-ATRP) allows the formation of a PNIPAAm interface with a well-defined densely packed

${ }^{a}$ Department of Materials Engineering, School of Engineering, The University of Tokyo, 7-3-1 Hongo, Bunkyo-ku, Tokyo 113-8656, Japan. E-mail: sakata@biofet.t.u-tokyo.ac. jp; ryo@cross.t.u-tokyo.ac.jp

${ }^{b}$ PROVIGATE Inc., The University of Tokyo, 7-3-1 Hongo, Bunkyo-ku, Tokyo 113-0033, Japan

'Institute of Advanced Biomiedical Engineering and Science, Tokyo Women's Medical University (TWIns), 8-1 Kawada-cho, Shinjuku-ku, Tokyo 162-8666, Japan

$\dagger$ Electronic supplementary information (ESI) available: Experimental procedures. See DOI: $10.1039 / \mathrm{c} 7 \mathrm{ra05786e}$

\$ Present address: School of Life Science and Technology, Tokyo Institute of Technology, 4259 Nagatsuta-cho, Midori-ku, Yokohama, Kanagawa 226-8501, Japan. face strongly interacts with biomolecules, leading to high efficiency in the separation of different biomolecules and cells by changing the PNIPAAm chain length, which can be precisely controlled by varying the monomer concentration in the process of SI-ATRP. ${ }^{11,15}$ Thus, the well-defined polymer film, whose thickness is precisely controlled by SI-ATRP, contributes to the detection of a quantitative signal by sensors and provides valuable information for the creation of novel bioanalytical devices.

Bioanalytical devices such as biosensors are being developed for the in situ monitoring of biological phenomena such as biomolecular recognition events and cellular activity. Recently, semiconductor-based biosensors have enabled the detection of biochemical functions in a real-time, label-free, and noninvasive manner based on the detection of changes in ionic charge density induced by biological phenomena. Field-effect transistor (FET)-based biosensing systems have been proposed for applications such as label-free DNA sequencing, ${ }^{16}$ immunological assay, ${ }^{17}$ saccharide sensing,${ }^{18}$ and cellular functional analysis. ${ }^{19,20}$ Such ionic charges around a biological target/ sensor interface must be selectively detected depending on the biological functions of interest. To analyze specific ionic behaviours on the basis of biological functions, therefore, an appropriate signal transduction material and structure should be developed around the biological target/sensor interface. By the grafting of functional groups on the surface of the gate, a specific target molecule can be detected quantitatively without labeling in real time. ${ }^{21}$ Furthermore, the volume phase transition of a glucose-responsive gel containing phenylboronic acid can be transduced into the electrical signal of a FET sensor, because a change in the permittivity of the gel grafted on the gate is induced by the dehydration of the gel. ${ }^{22,23}$ Therefore, the physicochemical phase transition of PNIPAAm by thermal 
stimulation should continuously be monitored as an electrical signal using the FET device, resulting in a strategy for designing and developing the signal transduction interface of biosensors.

In this study, we have clarified the dynamic electrical behaviour of a thermoresponsive PNIPAAm film on a FET device for the first time. As for the gate insulator of FET, $\mathrm{Ta}_{2} \mathrm{O}_{5}$ thin film was used as a passivation layer to prevent leakage currents in the solution. In addition, the ISFET with $\mathrm{Ta}_{2} \mathrm{O}_{5}$ insulator shows the high sensitivity for $\mathrm{pH}$ variation, approximately $60 \mathrm{mV} \mathrm{pH}^{-1}$ at $25^{\circ} \mathrm{C}$. In particular, the well-defined PNIPAAm, which was grafted on the $\mathrm{Ta}_{2} \mathrm{O}_{5}$ gate of a FET device by SI-ATRP, was characterized by, X-ray photoelectron spectroscopy (XPS). Using the PNIPAAm-grafted FET, we investigated whether the phase transition behaviour can be electrically detected. The temperature dependence of the electrical characteristics was evaluated for the well-defined PNIPAAm film grafted on the $\mathrm{Ta}_{2} \mathrm{O}_{5}$ gate, focusing on the changes in the gate surface potential and the capacitance.

Fig. 1 shows the schematic illustration for the modification process of PNIPAAm by ATRP on the $\mathrm{Ta}_{2} \mathrm{O}_{5}$ gate of FET device. At first, the ATRP initiators were modified at the $\mathrm{Ta}_{2} \mathrm{O}_{5}$ surface with hydroxyl groups by silane coupling method, then the PNIPAAm chains were grafted on it by the ATRP procedure (see experimental details in ESI $\dagger$ ). Table 1 shows the elemental composition of the prepared substrates was evaluated by XPS (the spectra are shown in and Fig. S1, in ESI†). The composition of carbon increased after immobilizing the ATRP initiator onto the $\mathrm{Ta}_{2} \mathrm{O}_{5}$ surface. After modifying PNIPAAm by SI-ATRP, hardly any tantalum, which originates from the gate insulator, was found, while the compositions of carbon and nitrogen increased on the $\mathrm{Ta}_{2} \mathrm{O}_{5}$ surface. Thus, PNIPAAm was successfully grafted on the $\mathrm{Ta}_{2} \mathrm{O}_{5}$ gate insulator of FET devices by the SIATRP procedure.

For the further characterization of PNIPAAm modified by ATRP, number-averaged molecular weight $\left(M_{\mathrm{n}}\right)$ polydispersity index (PDI) for the obtained free PNIPAAm were estimated by gel permeation chromatography (GPC) measurement (see experimental details in ESI $†$ ). An unbonded sacrificial ATRP initiator ( $\alpha$-chloro- $p$-xylene) was added to the reaction solution to obtain the free polymer because the previous study indicated that the molecular weight of the grafted polymer chain was similar to that of the free polymer. ${ }^{24}$ In addition, unbounded
Table 1 Elemental analyses by XPS ${ }^{a}$

\begin{tabular}{|c|c|c|c|c|c|c|c|}
\hline \multirow[b]{2}{*}{ Sample } & \multicolumn{5}{|c|}{ Atom (\%) } & \multirow[b]{2}{*}{$\mathrm{C} / \mathrm{Ta}$} & \multirow[b]{2}{*}{$\mathrm{N} / \mathrm{C}$} \\
\hline & $\mathrm{Ta}$ & $\mathrm{Cl}$ & $\mathrm{C}$ & $\mathrm{N}$ & $\mathrm{O}$ & & \\
\hline FET & 20.8 & n.d. & 16.2 & n.d. & 63.0 & 0.78 & - \\
\hline Initiator & 14.3 & 1.3 & 35.1 & n.d. & 49.3 & 2.5 & - \\
\hline PNIPAAm & 0.2 & n.d. & 76.1 & 11.8 & 11.9 & 380 & 0.16 \\
\hline
\end{tabular}

sacrificial initiator generates $\mathrm{Cu}^{\mathrm{II}} / \mathrm{Me}_{6} \mathrm{TREN}$ during ATRP, resulting in the control of polymerization. $M_{\mathrm{n}}$ and PDI were 2.6 $\times 10^{4}$ and 1.6, respectively. The PDI value was slightly large, which is because water markedly increased the polymerization rate in ATRP, ${ }^{25,26}$ but the ATRP procedure was more satisfactorily controlled than conventional free radical polymerization. Thus, the surface modification of PNIPAAm was quantitatively characterized. The additional characterization of the SI-ATRP conducted in this study are shown as Fig. S2 and S3 in ESI. $\dagger$

The phase transition of PNIPAAm induced by temperature changes was measured using the PNIPAAm-grafted FET device, as shown in Fig. 2 (the $I_{\mathrm{D}}-V_{\mathrm{G}}$ property of the FET device shown as Fig. S4 in ESI†). Fig. 2(a) shows the shift in the output voltage $\left(\Delta V_{\text {out }}\right)$ of non-grafted and PNIPAAm-grafted FETs with the change in the measurement temperature. $\Delta V_{\text {out }}$ at $20{ }^{\circ} \mathrm{C}$ was determined to be 0 . Water was used as the measurement solution. $\Delta V_{\text {out }}$ at the gate was measured at a constant $I_{\mathrm{D}}(700 \mu \mathrm{A})$ using the source follower circuit (Fig. S5 in ESI $\dagger$ ). The detected $\Delta V_{\text {out }}$ is regarded as the change in the source-gate voltage $\left(\Delta V_{\mathrm{S}}\right)$, which is equal to $-\Delta V_{\mathrm{T}}\left(\Delta V_{\mathrm{T}}\right.$ indicates the change in the threshold voltage). As the measurement temperature increased, $\Delta V_{\text {out }}$ gradually became increasingly negative for both FETs. This effect can be explained in terms of the isothermal point (zero-temperature-coefficient (ZTC) point) of FET. ${ }^{27}$ Basically, $I_{\mathrm{D}}$ strongly depends on the mobility and threshold voltage, which change with the temperature (see eqn (S6) in ESI†). The ZTC point is the bias point at which there is a mutual compensation between the temperature effects on the threshold voltage and mobility. That is, $\Delta V_{\text {out }}$ gradually shifted in the negative direction with increasing measurement temperature in Fig. 2 because of the temperature effect on the mobility at the

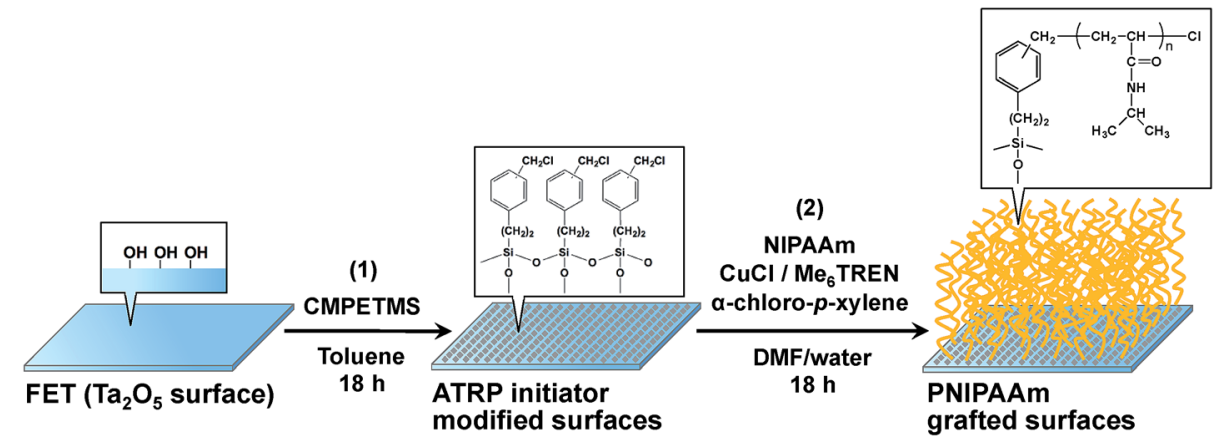

Fig. 1 Preparation of PNIPAAm surface onto the gate insulator of FET sensor. (1) Immobilization of ATRP initiator by silane coupling reaction. (2) SI-ATRP of PNIPAAm. 

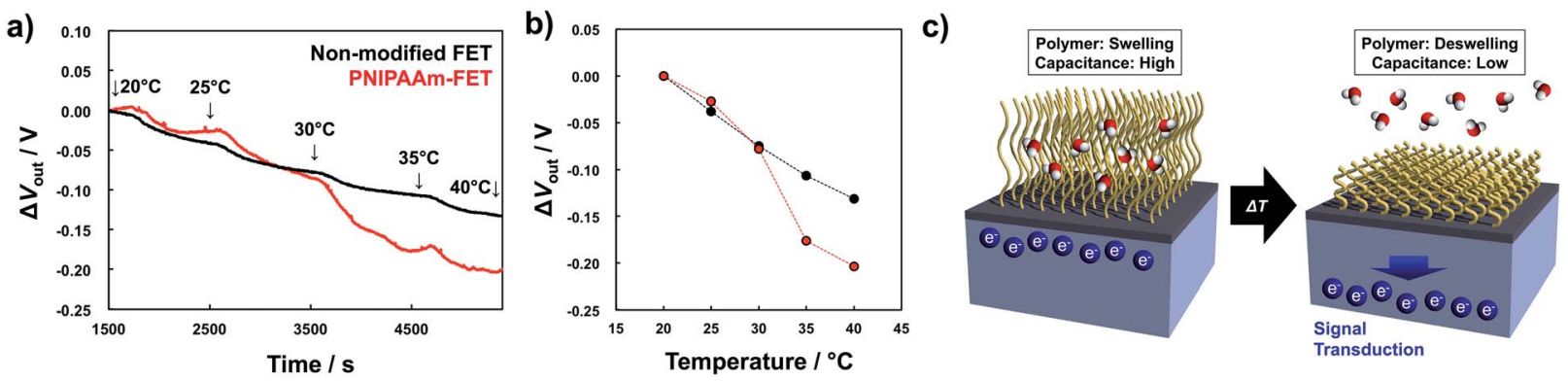

Fig. 2 (a) Real time monitoring of $\Delta V_{\text {out }}$ during the FET measurement for non-grafted FET (black line) and PNIPAAm grafted FET (red line). Temperature was changed from $20^{\circ} \mathrm{C}$ to $40^{\circ} \mathrm{C}$. (b) Temperature dependence of $\Delta V_{\text {out }}$ for non-grafted FET (black plot) and PNIPAAm grafted FET (red plot). (c) Illustration for the dynamic electrical behaviour of PNIPAAm-grafted FET.

constant $I_{\mathrm{D}}$ of $700 \mu \mathrm{A}$ (relatively high $I_{\mathrm{D}}$ ). In particular, $\Delta V_{\text {out }}$ of for the PNIPAAm-grafted FET became more negative than that of for the non-grafted FET, as the temperature increased from $30{ }^{\circ} \mathrm{C}$ to $35^{\circ} \mathrm{C}$, which corresponded to the LCST of PNIPAAm in water (Fig. 2(b)). For the PNIPAAm in water, phase transition from hydrated state to dehydrated state occurs across the LCST $\left(32{ }^{\circ} \mathrm{C}\right) .{ }^{9}$ Thus, it was indicated that a change in the physicochemical structure of PNIPAAm from swelling to deswelling was converted into a change in the electrical characteristic of FET device. The operation of a FET in the unsaturated region can generally be described by

$$
I_{\mathrm{D}}=\mu C_{\mathrm{OX}} \frac{W}{L}\left[\left(V_{\mathrm{G}}-V_{\mathrm{T}}\right) V_{\mathrm{D}}-\frac{1}{2} V_{\mathrm{D}}^{2}\right],
$$

where $I_{\mathrm{D}}$ is the drain current, $\mu$ is the electron mobility in the channel, $C_{\mathrm{OX}}$ is the gate oxide capacitance, $W / L$ is the channel width to channel length ratio, $V_{\mathrm{D}}$ and $V_{\mathrm{G}}$ are the applied drainsource and gate-source voltages, respectively, and $V_{\mathrm{T}}$ is the threshold voltage, which can be expressed by $y^{22,28}$

$$
V_{\mathrm{T}}=E_{\mathrm{ref}}-\psi_{0}+\chi^{\mathrm{sol}}-\frac{\phi_{\mathrm{si}}}{q}-\frac{Q_{\mathrm{it}}+Q_{\mathrm{f}}+Q_{\mathrm{B}}}{C_{\mathrm{OX}}}+2 \phi_{\mathrm{f}} .
$$

here, $E_{\text {ref }}$ is the reference electrode potential relative to a vacuum, $\left(-\psi_{0}+\chi^{\text {sol }}\right)$ describes the interfacial potential at the electrolyte/silicon dioxide interface (the factor $\chi^{\text {sol }}$ is the surface dipole moment of the solution, which can be considered to be constant), $\frac{\phi_{\mathrm{si}}}{q}$ is the electron work function of silicon, $Q_{\mathrm{it}}, Q_{\mathrm{f}}$, and $Q_{\mathrm{B}}$ are the charge of the interface traps, the fixed oxide charge, and the bulk depletion charge per unit area, respectively, and $\phi_{\mathrm{f}}$ is the Fermi potential difference between the doped bulk silicon and the intrinsic silicon.

Considering the capacitance of the PNIPAAm film grafted on the $\mathrm{Ta}_{2} \mathrm{O}_{5}$ gate insulator, eqn (2) is modified to

$$
V_{\mathrm{T}}=E_{\mathrm{ref}}-\psi_{0}+\chi^{\mathrm{sol}}-\frac{\phi_{\mathrm{si}}}{q}-\frac{Q_{\mathrm{it}}+Q_{\mathrm{f}}+Q_{\mathrm{B}}+Q_{\text {PNIPAAm }}}{C_{\text {total }}}+2 \phi_{\mathrm{f}}
$$

with

$$
C_{\text {total }}=\frac{C_{\mathrm{OX}} C_{\text {PNIPAAm }}}{C_{\mathrm{OX}}+C_{\text {PNIPAAm }}},
$$

where $Q_{\text {PNIPAAm }}$ is the charge in the PNIPAAm film and $C_{\text {total }}$ is the combined capacitance of $C_{\mathrm{OX}}$ and that of the PNIPAAm film $\left(C_{\text {PNIPAAm }}\right)$.

Here, $\psi_{0}$ is the actual driving force of the ion-sensitive FET (ISFET), which is related to the logarithm of the ion concentration. ${ }^{28}$ In this study, the physicochemical change in PNIPAAm on the gate was thermally induced without a change in ion concentrations; therefore, the capacitance of the polymer film on the gate should have mainly been altered, resulting in $\Delta V_{\text {out }}\left(-\Delta V_{\mathrm{T}}\right)$ in accordance with eqn (3) and (4). Actually, this means that the change in the capacitance of PNIPAAm-grafted FET causes the change in $I_{\mathrm{D}}\left(\Delta I_{\mathrm{D}}\right)$ as well as $\Delta V_{\mathrm{T}}$ according to eqn (1), unless $I_{\mathrm{D}}$ is constantly hold, as shown in Fig. 2(c).

To investigate the effect of a change in the capacitance of the polymer film on $\Delta V_{\text {out }}$ for the PNIPAAm-grafted FET device, $\mathrm{Ta}_{2} \mathrm{O}_{5}$-coated substrates were prepared with different surface modifications. Fig. 3 shows the temperature dependence of the capacitance for the non-grafted, ATRP initiator-immobilized, and PNIPAAm-grafted $\mathrm{Ta}_{2} \mathrm{O}_{5}$ substrates. As shown in Fig. 3(a) and (b), the capacitance for the ATRP initiator-immobilized $\mathrm{Ta}_{2} \mathrm{O}_{5}$ substrate was smaller than that of the non-grafted one; thus, a self-assembled monolayer (SAM) of the ATRP initiator was densely packed on the $\mathrm{Ta}_{2} \mathrm{O}_{5}$ substrate during the silane coupling reaction. After the modification of the PNIPAAm chain, moreover, the capacitance decreased owing to the increase in the thickness of the insulating layer, resulting in a change in its permittivity (Fig. 3(c)). Notably, the capacitances for the non-grafted and ATRP initiator-immobilized $\mathrm{Ta}_{2} \mathrm{O}_{5}$ substrates changed linearly with the measurement temperature (Fig. 3(a) and (b)), while the linearity was lost between $30^{\circ} \mathrm{C}$ and $35{ }^{\circ} \mathrm{C}$ for the PNIPAAm-grafted $\mathrm{Ta}_{2} \mathrm{O}_{5}$ substrate (Fig. $3(\mathrm{c})$ ). This is because although the capacitance generally increases with the measurement temperature, the dehydration of the PNIPAAm chain occurred around the LCST of $32{ }^{\circ} \mathrm{C}$. That is, the PNIPAAm chain film changed to the dehydrated state above the LCST, resulting in a decrease in its permittivity (the relative permittivity of water is approximately 80 at $20{ }^{\circ} \mathrm{C}$ ). The dehydration above the LCST was also confirmed by the impedance measurement for the PNIPAAm-grafted $\mathrm{Ta}_{2} \mathrm{O}_{5}$ substrate (Fig. S6 and S7 in ESI + ). Thus, the phase transition of PNIPAAm contributed to the loss of linearity across the LCST, causing the change in the capacitance of the PNIPAAm-grafted $\mathrm{Ta}_{2} \mathrm{O}_{5}$ 

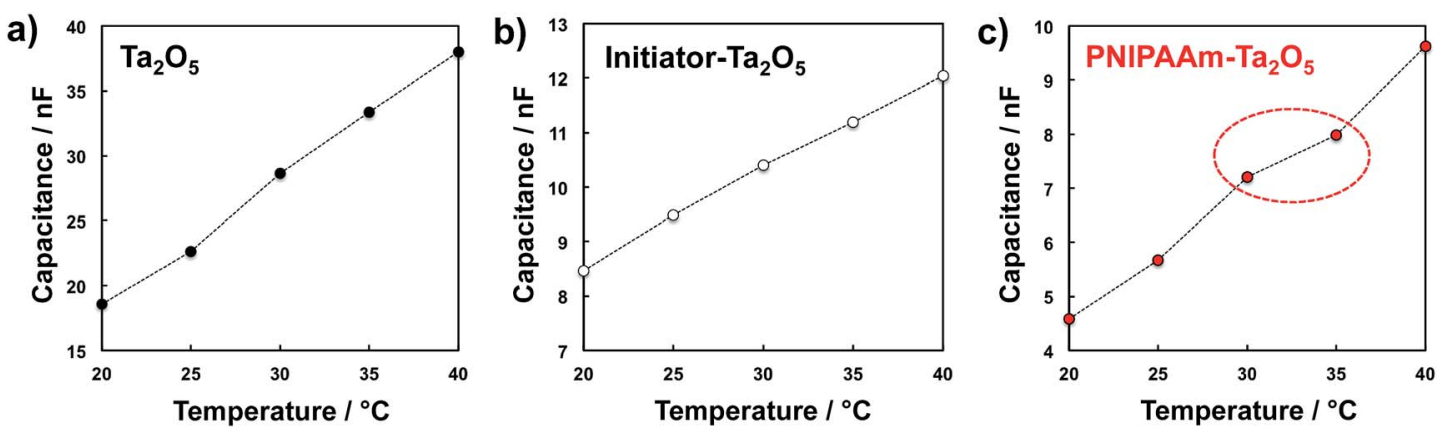

Fig. 3 Temperature dependence of the capacitances for (a) non-modified $\mathrm{Ta}_{2} \mathrm{O}_{5}$ substrate, (b) ATRP initiator-modified $\mathrm{Ta}_{2} \mathrm{O}_{5}$ substrate and (c) PNIPAAm-modified $\mathrm{Ta}_{2} \mathrm{O}_{5}$ substrate.

substrate, which shifted in the negative direction owing to a change from swelling to deswelling.

According to eqn (3) and (4), the FET biosensor responds to changes in the ionic concentrations and capacitances of interfacial materials around a solution/gate interface resulting from biological phenomena. In fact, an ISFET sensor is based on the potentiometric detection of changes in the concentration of ions such as hydrogen ions $(\mathrm{pH})$, which are detected by the interaction with hydroxyl groups at the oxide gate surface on the basis of the equilibrium reaction. ${ }^{28}$ The electrical signal generated by $\mathrm{pH}$ variation is evaluated as $\Delta V_{\mathrm{T}}$, which depends on the change in $\psi_{0}\left(\Delta \psi_{0}\right)$ in eqn (3), resulting in Nernstian response $\left(59.1 \mathrm{mV} \mathrm{pH}^{-1}\right.$ at $\left.25^{\circ} \mathrm{C}\right)$. On the other hand, the FET biosensor can recognize changes in the capacitance of functional materials on the gate surface such as the thermoresponsive polymer in this study. As shown in Fig. 2(b), the difference in $\Delta V_{\text {out }}$ between the PNIPAAm-grafted FET and the non-grafted FET (thermally responsive signal) was larger than Nernstian response based on the change in the ion concentrations, which was approximately $75 \mathrm{mV}$ above $35^{\circ} \mathrm{C}$ owing to the dehydration of PNIPAAm across the LCST. This means that the output signal of the FET biosensor can be amplified by functionalization with interface materials that exhibit a phase transition upon strongly and selectively interacting with ions and biomolecules or upon thermal stimulation. Moreover, the well-defined polymer film with controlled thickness obtained by ATRP should provide significant information for quantitative analyses with biosensors. The target molecule can be detected by copolymerizing molecular recognition site to the polymer brush. For the application of the polymer brush-grafted FET, reversibility of this system and the relationship between the structure of the polymer brush and the electrical behaviour in FET are important. These attempts are currently being investigated. Thus, our findings on the signal transduction of physicochemical behaviour of polymer to electrical property in this study will be helpful for the design, control, and development of novel biosensing devices.

\section{Conclusions}

In summary, we have investigated how the physicochemical behaviour of the thermoresponsive polymer PNIPAAm, such as swelling and deswelling, is transduced into an electronic signal by a FET sensor. In particular, a well-defined polymer film with controlled thickness was grafted on the $\mathrm{Ta}_{2} \mathrm{O}_{5}$ gate of a FET device by SI-ATRP to quantitatively analyze the electrical characteristics of the polymer film. As the measurement temperature increased, $\Delta V_{\text {out }}$ for the PNIPAAm-grafted FET clearly shifted in the negative direction as a result of the physicochemical change from swelling to deswelling across the LCST, compared with the non-grafted FET. The signal shift was mainly caused by the change in the capacitance of the thermoresponsive polymer grafted on the gate. Basically, $\Delta V_{\mathrm{T}}\left(-\Delta V_{\text {out }}\right)$ for the FET sensor strongly depends on the change in the ion concentration around a solution/gate interface and the capacitance of the functional interface materials on the gate; therefore, the output signal of the FET biosensor can be amplified by functionalization with interface materials that exhibit a phase transition upon strongly and selectively interacting with ions and biomolecules or upon thermal stimulations. We believe that this work will significantly contribute to the design, control, and development of signal transduction interfaces for novel biosensing devices.

\section{Acknowledgements}

This work was supported in part by a Grant-in-Aid for Scientific Research from the Ministry of Education, Culture, Sports, Science and Technology of Japan (Grant No. 15H02198 to R. Y.).

\section{Notes and references}

1 T. Wu, Y. Zhang, X. Wang and S. Liu, Chem. Mater., 2008, 20, 101.

2 A. Kopyshev, C. J. Galvin, R. R. Patil, J. Genzer, N. Lomadze, D. Feldmann, J. Zakrevski and S. Santer, ACS Appl. Mater. Interfaces, 2016, 8, 19175.

3 A. J. Parnell, S. J. Martin, R. A. L. Jones, C. Vasilev, C. J. Crook and A. J. Ryan, Soft Matter, 2009, 5, 296.

4 S. Kumar, X. Tong, Y. L. Dory, M. Lepage and Y. Zhao, Chem. Commun., 2013, 49, 90.

5 Y. Zou, A. Lam, D. E. Brooks, A. S. Phani and J. N. Kizhakkedathu, Angew. Chem., Int. Ed., 2011, 50, 5116. 
6 G. J. Dunderdale, C. Urata and A. Hozumi, Langmuir, 2014, 30, 13438.

7 Q. Wei, M. Cai, F. Zhou and W. Liu, Macromolecules, 2013, 46, 9368.

8 M. Szuwarzynski, L. Zaraska, G. D. Sulka and S. Zapotoczny, Chem. Mater., 2013, 25, 514.

9 M. Heskins and J. E. Guillet, J. Macromol. Sci., Part A: Pure Appl. Chem., 1968, 2, 1441.

10 N. Idota, A. Kikuchi, J. Kobayashi, K. Sakai and T. Okano, Adv. Mater., 2005, 17, 2723.

11 K. Nagase, J. Kobayashi, A. Kikuchi, Y. Akiyama, H. Kanazawa and T. Okano, Langmuir, 2007, 23, 9409.

12 N. Matuzaka, M. Nakayama, H. Takahashi, M. Yamato, A. Kikuchi and T. Okano, Biomacromolecules, 2013, 14, 3164.

13 Y. Akiyama, A. Kikuchi, M. Yamato and T. Okano, Langmuir, 2004, 20, 5506.

14 T. Yakushiji, K. Sakai, A. Kikuchi, T. Aoyagi, Y. Sakurai and T. Okano, Langmuir, 1998, 14, 4657.

15 K. Nagase, A. Kimura, T. Shimuzu, K. Matsuura, M. Yamato, N. Takeda and T. Okano, J. Mater. Chem., 2012, 22, 19514.

16 T. Sakata and Y. Miyahara, Angew. Chem., Int. Ed., 2006, 45, 2225.
17 T. Sakata, M. Ihara, I. Makino, Y. Miyahara and H. Ueda, Anal. Chem., 2009, 81, 7532.

18 T. Kajisa and T. Sakata, ChemElectroChem, 2014, 1, 1647.

19 P. Fromherz, A. Offenhausser, T. Vetter and K. Weis, Science, 1991, 252, 1290.

20 T. Sakata, A. Saito, J. Mizuno, H. Sugimoto, K. Noguchi, E. Kikuchi and H. Inui, Anal. Chem., 2013, 85, 6633.

21 H. Nishida, T. Kajisa, Y. Miyazawa, Y. Tabuse, T. Yoda, H. Takeyama, H. Kambara and T. Sakata, Langmuir, 2015, 31, 732 .

22 A. Matsumoto, N. Sato, T. Sakata, R. Yoshida, K. Kataoka and Y. Miyahara, Adv. Mater., 2009, 21, 4372.

23 A. Matsumoto, T. Endo, R. Yoshida and Y. Miyahara, Chem. Commun., 2009, 5609.

24 M. Ejaz, S. Yamamoto, K. Ohno, Y. Tsujii and T. Fukuda, Macromolecules, 1998, 31, 5934.

25 D. M. l. Jones and W. T. S. Huck, Adv. Mater., 2001, 13, 1256. 26 K. Nagase, A. M. Akimoto, J. Kobayashi, A. Kikuchi, Y. Akiyama, H. Kanazawa and T. Okano, J. Chromatogr. A, 2011, 1218, 8617.

27 P. Toledo, H. Klimach, D. Cordova, S. Bampi and E. Fabris, Journal of Integrated Circuits and Systems, 2015, 10, 103.

28 P. Bergveld, Sens. Actuators, 1985, 8, 109. 